hep-th/0101221

\title{
Black diholes with unbalanced magnetic charges
}

\author{
Y.C. Liang and Edward Teo \\ Department of Physics \\ National University of Singapore \\ Singapore 119260
}

\begin{abstract}
We present a technique that can be used to generate a static, axisymmetric solution of the Einstein-Maxwell-Dilaton equations from a stationary, axisymmetric solution of the vacuum Einstein equations. Starting from the Kerr solution, Davidson and Gedalin have previously made use of this technique to obtain a pair of oppositely charged, extremal dilatonic black holes, known as a black dihole. In this paper, we shall instead start from the Kerr-NUT solution. It will be shown that the new solution can also be interpreted as a dihole, but with the black holes carrying unbalanced magnetic charges. The effect of the NUT-parameter is to introduce a net magnetic charge into the system. Finally, we uplift our solution to ten dimensions to describe a system consisting of $D 6$ and anti-D6-branes with unbalanced charges. The limit in which they coincide agrees with a solution recently derived by Brax et al..
\end{abstract}




\section{Introduction}

In 1983, Gross and Perry [1] and Sorkin [2] derived solutions of Kaluza-Klein theory starting from existing solutions to the four-dimensional vacuum Einstein equations. These solutions were constructed by first analytically continuing the seed solutions to the Euclidean regime $t \rightarrow i x^{5}$, and adding on an extra flat time direction. Solutions to the four-dimensional Kaluza-Klein equations can then be obtained by compactifying the five-dimensional spacetimes on $x^{5}$.

Using the self-dual Taub-NUT solution as seed, the authors [1, \#] obtained a static solution whose Kaluza-Klein gauge field corresponds to that of a magnetic monopole. This gauge field originates from the $g_{t \varphi}$ term of the Taub-NUT solution, with the NUT-parameter attributed the physical meaning of magnetic charge in the new solution.

Similarly, by using the Kerr solution as seed, a static solution whose gauge field describes that of a magnetic dipole was constructed [1]. In this case, the angular momentum of the Kerr solution is converted into a parameter that characterizes the dipole moment of the new solution. The latter is the Kaluza-Klein analog of the Bonnor solution [3] which describes a magnetic dipole in general relativity.

Now, Kaluza-Klein theory is a special case of the more general Einstein-Maxwell-Dilaton theory with arbitrary dilatonic coupling. In the former, the coupling constant $\alpha$, defined in (2.1), is taken to be $\sqrt{3}$. However, in this paper, we shall focus on the more general EinsteinMaxwell-Dilaton theory where $\alpha$ can take on any real value.

In 1994, Davidson and Gedalin [团] generalized the solution-generating technique to one that is valid for arbitrary dilatonic coupling using a Ernst-type formalism. Starting from the Kerr solution, they obtained the dilatonic generalization of the Bonnor solution and interpreted the $\alpha=1$ case as one exhibiting a two-dimensional black and white dihole structure. However, Emparan [5] recently pointed out a flaw in this interpretation and demonstrated that the solution (for general $\alpha$ ) actually describes a static pair of oppositely charged, extremal dilatonic black holes, which he calls a black dihole.

We will start off in Sec. 2 by presenting the solution-generating technique that was first used by Davidson and Gedalin to obtain the dilatonic generalization of the Bonnor solution. Since this technique was only briefly described in their original article [ब, we will provide details of it for completeness. To the best of our knowledge, these details cannot be found elsewhere in the literature.

In Sec. 3, we will make use of this technique to derive our dihole solution starting from the Kerr-NUT solution [6]; the latter is a generalization of the Kerr solution which includes 
an additional NUT-parameter. As such, our solution contains all the above-mentioned ones as special cases.

This will be followed by a standard analysis, that was first used by Emparan [5], to investigate the physical nature of the new solution. We will demonstrate that it describes a pair of extremal dilatonic black holes carrying unbalanced magnetic charges, i.e. each of the black holes carries a magnetic charge that is of different sign as well as magnitude. In contrast to a dipole, this system will have a non-trivial net magnetic charge. We will continue to refer to this solution as a dihole.

In Sec. 4, we will discuss the embedding of our solution in Type IIA superstring theory, in which it would describe a static configuration of $D 6$ and anti- $D 6$-branes with unbalanced charges. We will then show that the coincident limit agrees with a solution recently presented by Brax et al. [7].

\section{Solution-generating technique}

The idea of generating a solution of the Einstein-Maxwell equations starting from a solution of the vacuum Einstein equations is not a new one (see for example Kramer et al. [8] or Islam [9]). In this section, we will focus on a different technique which generates a static, axisymmetric solution of the Einstein-Maxwell-Dilaton equations starting from a stationary, axisymmetric solution of the vacuum Einstein equations.

Einstein-Maxwell-Dilaton theory in four dimensions has the following action integral:

$$
\int \mathrm{d}^{4} x \sqrt{-g}\left(R-2(\nabla \phi)^{2}-e^{-2 \alpha \phi} F^{2}\right)
$$

where $R$ is the Ricci scalar, $\phi$ the dilaton field and $F_{a b}$ the electromagnetic field tensor. For the special case $\alpha=0$, we recover Einstein-Maxwell theory; for $\alpha=1$, the action describes the low energy dynamics of string theory; and for $\alpha=\sqrt{3}$, we have Kaluza-Klein theory as mentioned in the introduction. However, we shall keep $\alpha$ general in what follows. By varying this action with respect to the metric, gauge field and dilaton field, we obtain the respective field equations:

$$
\begin{aligned}
& R_{a b}=2 \nabla_{a} \phi \nabla_{b} \phi+e^{-2 \alpha \phi}\left(2 F_{a c} F_{b}^{c}-\frac{1}{2} g_{a b} F^{2}\right) \\
& \nabla_{a}\left(e^{-2 \alpha \phi} F^{a b}\right)=0 \\
& \nabla^{2} \phi+\frac{\alpha}{2} e^{-2 \alpha \phi} F^{2}=0 .
\end{aligned}
$$


Now, we are looking for a static, axisymmetric solution to the field equations. Recall that any such spacetime can be cast in the Weyl-Papapetrou form 9]:

$$
\mathrm{d} s^{2}=-f \mathrm{~d} t^{2}+l \mathrm{~d} \varphi^{2}+e^{\mu}\left(\mathrm{d} \rho^{2}+\mathrm{d} z^{2}\right) .
$$

Furthermore, we choose a purely magnetic ansätz $A \equiv A_{\varphi}$ with all other components of $A_{a}$ vanishing. It is also understood that $f, l, \mu, A$ and $\phi$ are functions of $\rho$ and $z$ only.

With this ansätz, we can now evaluate the left- and right-hand sides of 2.2a). Defining $D^{2} \equiv f l$, and by considering the combination $e^{\mu} D^{-1}\left(l R_{t t}-f R_{\varphi \varphi}\right)$, we obtain

$$
\frac{\partial^{2} D}{\partial \rho^{2}}+\frac{\partial^{2} D}{\partial z^{2}}=0
$$

A simple solution to this equation is given by $D=\rho$ [9]. With this choice, we may evaluate the $R_{t t}$ equation to arrive at

$$
f\left(f_{\rho \rho}+f_{z z}+\rho^{-1} f_{\rho}\right)-f_{\rho}^{2}-f_{z}^{2}=2 \rho^{-2} f^{3} e^{-2 \alpha \phi}\left(A_{\rho}^{2}+A_{z}^{2}\right),
$$

where subscripts $\rho$ and $z$ indicate partial derivatives of the corresponding function with respect to these variables. Similarly, by evaluating the Ricci components $R_{\rho \rho}-R_{z z}$ and $R_{\rho z}$, we obtain respectively

$$
\begin{aligned}
& \mu_{\rho}=-f^{-1} f_{\rho}+\frac{1}{2} \rho f^{-2}\left(f_{\rho}{ }^{2}-f_{z}{ }^{2}\right)+2 \rho\left(\phi_{\rho}{ }^{2}-\phi_{z}{ }^{2}\right)+2 e^{-2 \alpha \phi} \rho^{-1} f\left(A_{\rho}{ }^{2}-A_{z}{ }^{2}\right), \\
& \mu_{z}=-f^{-1} f_{z}+\rho f^{-2} f_{\rho} f_{z}+4 \rho \phi_{\rho} \phi_{z}+4 e^{-2 \alpha \phi} \rho^{-1} f A_{\rho} A_{z} .
\end{aligned}
$$

Finally, we obtain from $(2.2 \mathrm{~b})$ and $(2.2 \mathrm{~g})$ the gauge field and dilaton equations respectively:

$$
\begin{aligned}
& A_{\rho \rho}+A_{z z}-\rho^{-1} A_{\rho}=2 \alpha\left(A_{\rho} \phi_{\rho}+A_{z} \phi_{z}\right)-f^{-1}\left(A_{\rho} f_{\rho}+A_{z} f_{z}\right), \\
& \phi_{\rho \rho}+\phi_{z z}+\rho^{-1} \phi_{\rho}=-\alpha \rho^{-2} f e^{-2 \alpha \phi}\left(A_{\rho}^{2}+A_{z}^{2}\right) .
\end{aligned}
$$

The crucial step now is to realize that if we set

$$
\tilde{f}^{2}=f e^{-2 \alpha \phi} \quad \text { and } \quad w=i \sqrt{1+\alpha^{2}} A,
$$

then (2.4) and (2.6) respectively become

$$
\begin{gathered}
\tilde{f}\left(\tilde{f}_{\rho \rho}+\tilde{f}_{z z}+\rho^{-1} \tilde{f}_{\rho}\right)-\tilde{f}_{\rho}{ }^{2}-\tilde{f}_{z}{ }^{2}+\rho^{-2} \tilde{f}^{4}\left(w_{\rho}{ }^{2}+w_{z}{ }^{2}\right)=0, \\
\tilde{f}\left(w_{\rho \rho}+w_{z z}-\rho^{-1} w_{\rho}\right)+2\left(w_{\rho} \tilde{f}_{\rho}+w_{z} \tilde{f}_{z}\right)=0 .
\end{gathered}
$$


These equations are precisely the same as those derived from the vacuum Einstein equations for a stationary, axisymmetric metric

$$
\mathrm{d} s^{2}=-\tilde{f}(\mathrm{~d} t-w \mathrm{~d} \varphi)^{2}+\rho^{2} \tilde{f}^{-1} \mathrm{~d} \varphi^{2}+e^{\tilde{\mu}}\left(\mathrm{d} \rho^{2}+\mathrm{d} z^{2}\right)
$$

using a Ernst-type formalism (c.f. Eqn. (2.12a) and (2.12b) of Islam [9]). For every such solution to the vacuum Einstein equations, we can therefore find a corresponding solution to the Einstein-Maxwell-Dilaton equations via (2.8). Nevertheless, one should be aware that this procedure would in general generate an imaginary gauge field. Thus, a real solution to the Einstein-Maxwell-Dilaton equations can be generated via this method only if an analytic continuation of the parameter(s) in the seed solution is possible.

Finally, we see that the dilaton equation (2.7) can be written as

$$
\phi_{\rho \rho}+\phi_{z z}+\rho^{-1} \phi_{\rho}=-\alpha \rho^{-2} \tilde{f}^{2}\left(A_{\rho}^{2}+A_{z}^{2}\right)
$$

which, using (2.9), admits the solution

$$
\phi=-\frac{\alpha}{1+\alpha^{2}} \ln \tilde{f}
$$

up to the addition of a harmonic function $\tilde{\phi}$ satisfying $\tilde{\phi}_{\rho \rho}+\tilde{\phi}_{z z}+\rho^{-1} \tilde{\phi}_{\rho}=0$. For the choice $\tilde{\phi}=0$, we have

$$
f=\tilde{f}^{\frac{2}{1+\alpha^{2}}} .
$$

These expressions, together with that for the gauge field obtained from (2.8), can then be used to deduce $\mu$ via (2.5a) and (2.5b). This completes our derivation of the static, axisymmetric solution to the field equations (2.2a $)-(2.2 \mathrm{a})$.

We remark that this technique is just a dilatonic generalization of Theorem 30.8 in Kramer et al. [8], which Bonnor [3] used to generate (from the Kerr solution) the wellknown magnetic dipole solution. Using the same solution as seed, Davidson and Gedalin [4] have made use of the above technique to generate the dilatonic generalization of the Bonnor solution. If we begin with the self-dual Taub-NUT solution instead, we would obtain a generalization of the Gross-Perry-Sorkin [1, 2] monopole solution to arbitrary dilatonic coupling, which can also be interpreted as an extremal dilatonic black hole [10]. 


\section{Dihole solution with unbalanced charges}

\subsection{Derivation of solution}

In this paper, we will start from the Kerr-NUT (or Demiański-Newman) solution [6]:

$$
\begin{aligned}
\mathrm{d} s^{2}= & -\bar{\Lambda}\left(\mathrm{d} t+2 \frac{a \sin ^{2} \theta\left(m r+l^{2}\right)+l \bar{\Delta} \cos \theta}{\bar{\Delta}-a^{2} \sin ^{2} \theta} \mathrm{d} \varphi\right)^{2} \\
& +\bar{\Lambda}^{-1}\left[\left(\bar{\Delta}-a^{2} \sin ^{2} \theta\right)\left(\frac{\mathrm{d} r^{2}}{\bar{\Delta}}+\mathrm{d} \theta^{2}\right)+\bar{\Delta} \sin ^{2} \theta \mathrm{d} \varphi^{2}\right]
\end{aligned}
$$

where

$$
\bar{\Lambda} \equiv \frac{\bar{\Delta}-a^{2} \sin ^{2} \theta}{r^{2}+(a \cos \theta+l)^{2}} \quad \text { and } \quad \bar{\Delta} \equiv r^{2}-2 m r+a^{2}-l^{2} .
$$

In these expressions, $a$ is the angular momentum and $l$ is the NUT-parameter. Note that this solution contains both the Kerr and Taub-NUT solutions as special cases with $l=0$ and $a=0$ respectively.

By comparing the line element (3.1) with (2.11), we obtain

$$
\tilde{f}=\bar{\Lambda} \quad \text { and } \quad w=-2 \frac{a \sin ^{2} \theta\left(m r+l^{2}\right)+l \bar{\Delta} \cos \theta}{\bar{\Delta}-a^{2} \sin ^{2} \theta} .
$$

To ensure that the resulting gauge field is real, we perform the analytic continuation $a \rightarrow i a$ and $l \rightarrow i l$. Using (2.8), (2.13) and (2.14), we obtain

$$
\begin{aligned}
A_{\varphi} & =-\frac{2}{\sqrt{1+\alpha^{2}}} \frac{a \sin ^{2} \theta\left(m r-l^{2}\right)+l \Delta \cos \theta}{\Delta+a^{2} \sin ^{2} \theta}, \\
\phi & =-\frac{\alpha}{1+\alpha^{2}} \ln \left[\frac{\Delta+a^{2} \sin ^{2} \theta}{\Sigma}\right], \\
f & =\left[\frac{\Delta+a^{2} \sin ^{2} \theta}{\Sigma}\right]^{\frac{2}{1+\alpha^{2}}},
\end{aligned}
$$

where

$$
\Delta \equiv r^{2}-2 m r-a^{2}+l^{2} \quad \text { and } \quad \Sigma \equiv r^{2}-(a \cos \theta+l)^{2} .
$$

Substituting (3.3) - (3.5) into (2.5a) and (2.5b), and by transforming from the BoyerLindquist-type coordinates $(r, \theta)$ to cylindrical coordinates $(\rho, z)$ via 11

$$
\rho=\sqrt{r^{2}-2 m r-a^{2}+l^{2}} \sin \theta, \quad z=(r-m) \cos \theta
$$


we obtain by quadraturef the expression for $\mu$. We finally arrive at the new solution

$$
\mathrm{d} s^{2}=\Lambda^{\frac{2}{1+\alpha^{2}}}\left\{-\mathrm{d} t^{2}+\frac{\sum^{\frac{4}{1+\alpha^{2}}}}{\left[\Delta+\left(m^{2}+a^{2}-l^{2}\right) \sin ^{2} \theta\right]^{\frac{3-\alpha^{2}}{1+\alpha^{2}}}}\left(\frac{\mathrm{d} r^{2}}{\Delta}+\mathrm{d} \theta^{2}\right)\right\}+\frac{\Delta \sin ^{2} \theta}{\Lambda^{\frac{2}{1+\alpha^{2}}}} \mathrm{~d} \varphi^{2}
$$

where

$$
\Lambda \equiv \frac{\Delta+a^{2} \sin ^{2} \theta}{\Sigma}
$$

with $A_{\varphi}$ and $\phi$ given in (3.3) and (3.4) respectively.

\subsection{Physical properties of the solution}

Recently, the special case $(l=0)$ of the above solution was analyzed in detail by Emparan [5]. In this section, we will perform a similar analysis on our solution and arrive at the conclusion that it describes a pair of extremal dilatonic black holes with unbalanced charges lying on the symmetry axis.

We begin by highlighting that, in addition to being static and axisymmetric, the solution is asymptotically flat. This is in contrast to our seed solution (3.1) whose $g_{t \varphi}$ term does not vanish in the asymptotic limit. This unphysical nature of the Kerr-NUT solution has thus been removed in the new solution.

A study of the asymptotic behavior of $g_{t t}$ and $A_{\varphi}$ also reveals that the total mass of the solution is $M=\frac{2 m}{1+\alpha^{2}}$ whereas the net magnetic charge of the solution is $\bar{Q}=\frac{2 l}{\sqrt{1+\alpha^{2}}}$. Thus the NUT-parameter $l$ governs the monopole field strength of the solution at far field. Without loss of generality, we shall restrict ourselves to non-negative $l$ corresponding to non-negative net magnetic charge.

We shall now examine the singularities of the metric. By evaluating the curvature invariant $R_{a b c d} R^{a b c d}$, it can be checked that for $l \leq m$ i, the "outermost" curvature singularities are located at the two points:

$$
r=r_{+} \equiv m+\sqrt{m^{2}+a^{2}-l^{2}} \quad, \quad \theta=0, \pi .
$$

${ }^{*}$ For practical reasons, the integration was actually performed after further transforming to prolate spheroidal coordinates [1]]:

$$
x=\frac{r-m}{\sqrt{m^{2}+a^{2}-l^{2}}} \quad \text { and } \quad y=\cos \theta
$$

${ }^{\dagger}$ This range for $l$ will be justified below. 
We can then follow a similar analysis as in [5] to show that the axis of symmetry consists of the three line segments $\theta=0, r=r_{+}$and $\theta=\pi$, and the singularities given by (3.8) are merely the joints between these segments (c.f. Fig 1 of [5]).

In order to better understand the nature of these singularities, we first note that the proper distance between the two singularities increases as $2 a$ when $a \rightarrow \infty$. It can also be shown that (for $\alpha \neq 0$ ) the proper distance vanishes when $a \rightarrow 0 \in$ Thus, the parameter $a$ serves as a measure of the distance between the two singularities.

Bearing these facts in mind, we may now further investigate the two singularities by adopting the following transformation [12, 5]:

$$
r=r_{+}+\frac{\tilde{r}}{2}(1+\cos \tilde{\theta}) \quad \text { and } \quad \sin ^{2} \theta=\frac{\tilde{r}(1-\cos \tilde{\theta})}{\sqrt{m^{2}+a^{2}-l^{2}}}
$$

on the metric (3.7), while taking the limit $a \rightarrow \infty$. Physically, this is tantamount to pushing one of the singularities to a large distance and studying the geometry of the remaining singularity. After carrying out the transformation, we obtain

$$
\begin{aligned}
\mathrm{d} s^{2} & \rightarrow-\left(1+\frac{|Q|}{\tilde{r}}\right)^{-\frac{2}{1+\alpha^{2}}} \mathrm{~d} t^{2}+\left(1+\frac{|Q|}{\tilde{r}}\right)^{\frac{2}{1+\alpha^{2}}}\left[\mathrm{~d} \tilde{r}^{2}+\tilde{r}^{2}\left(\mathrm{~d} \tilde{\theta}^{2}+\sin ^{2} \tilde{\theta} \mathrm{d} \varphi^{2}\right)\right] \\
A_{\varphi} & \rightarrow \frac{Q \cos \tilde{\theta}}{\sqrt{1+\alpha^{2}}} \\
\phi & \rightarrow-\frac{\alpha}{1+\alpha^{2}} \ln \left(1+\frac{|Q|}{\tilde{r}}\right),
\end{aligned}
$$

where

$$
\left.Q\right|_{\theta=0}=m-l \quad \text { and }\left.\quad Q\right|_{\theta=\pi}=-m-l .
$$

This limiting form is just that of an extremal dilatonic black hole, with the (singular) horizon located at $\tilde{r}=0\left(r=r_{+}\right)$[10].

We could also perform the transformation (3.9) on the metric (3.7) without taking the limit of large $a$. For small $\tilde{r}$, it enables us to investigate the geometry near to the two singularities. In this limit, the geometry reduces to the near-horizon limit of an extremal dilatonic black hole. However, the horizon will no longer be spherically symmetric due to the presence of the other black hole. One can readily calculate the relevant distortion factors following [5].

\footnotetext{
${ }^{\ddagger}$ For $\alpha=0$ however, the proper distance remains infinite in this limit. It would be clear later that this is due to the well-known fact that extremal Reissner-Nordström black holes have throats of infinite length.
} 
It can therefore be seen that at the ends of the segment $r=r_{+}$, there lie two extremal dilatonic black holes carrying unbalanced magnetic charges. With the aid of Gauss's law, we may also deduce from (3.10b) that the black hole at $(r, \theta)=\left(r_{+}, 0\right)$ and $\left(r_{+}, \pi\right)$ carries a magnetic charge of $\frac{l-m}{\sqrt{1+\alpha^{2}}}$ and $\frac{l+m}{\sqrt{1+\alpha^{2}}}$ respectively. As expected, the sum of these magnetic charges matches exactly with the net charge of the solution obtained above.

The next step is to determine if there are any conical singularities along the different segments of the symmetry axis. Assuming that the coordinate $\varphi$ has its usual periodicity along the symmetry axes $\theta=0$ or $\pi$, it can be checked that the conical excess along $r=r_{+}$ is given by

$$
\delta_{\left(r_{+}\right)}=2 \pi\left[\left(1+\frac{m^{2}-l^{2}}{a^{2}}\right)^{\frac{2}{1+\alpha^{2}}}-1\right] .
$$

As was pointed out in [5], this conical excess can be understood physically as the presence of a strut along $r=r_{+}$, which provides the necessary internal stress to counterbalance the attraction between the unbalanced-charged black holes.

To an observer located at $r>r_{+}$, the only observable physical entities are thus the two black holes located at the ends of the segment $r=r_{+}$; when $0<\theta<\pi$, the region $r<r_{+}$is inaccessible due to the presence of conical singularities; when $\theta=0$ or $\pi$, all other singularities are located at $r<r_{+}$, i.e. enclosed within the horizon.

Now, note from (3.10d) and (3.11) that the magnetic charge of the black hole at $\left(r_{+}\right.$, 0 ), as well as the conical singularity along the segment $r=r_{+}$, vanishes when $l=m$. To understand the physical nature of this special case, we first note from (3.10a) that the masses of the black holes are $\left.m\right|_{\theta=0}=\frac{m-l}{1+\alpha^{2}}$ and $\left.m\right|_{\theta=\pi}=\frac{m+l}{1+\alpha^{2}}$ respectively. When $l=m$, the mass of the black hole at $\theta=0$ vanishes whereas that of the one at $\theta=\pi$ becomes the total mass of the solution. Intuitively, we can thus think of the increase of $l$ (from zero) as a physical process whereby the mass 8 of the first black hole is transferred adiabatically to the second.

Indeed, this can seen by performing the following transformation on the line element (3.7) when $l=m$ :

$$
\tilde{r}=r-m+a \cos \theta \quad \text { and } \quad \sin ^{2} \tilde{\theta}=\frac{(r-m)^{2}-a^{2}}{(r-m+a \cos \theta)^{2}} \sin ^{2} \theta .
$$

The resulting line element is

$$
\mathrm{d} s^{2}=-\left(1+\frac{2 m}{\tilde{r}}\right)^{-\frac{2}{1+\alpha^{2}}} \mathrm{~d} t^{2}+\left(1+\frac{2 m}{\tilde{r}}\right)^{\frac{2}{1+\alpha^{2}}}\left[\mathrm{~d} \tilde{r}^{2}+\tilde{r}^{2}\left(\mathrm{~d} \tilde{\theta}^{2}+\sin ^{2} \tilde{\theta} \mathrm{d} \varphi^{2}\right)\right],
$$

\footnotetext{
${ }^{\S}$ In the process of increasing the value of $l$, charge is being transferred as well since the black holes are extremal.
} 
which clearly describes the geometry of an extremal dilatonic black hole with mass $2 m$ (c.f. (3.10a)). Note that although the transformation depends on $a$, the resulting line element does not; this is expected because $a$ no longer carries any physical meaning when there is only one black hole left in the system. Notice that if we attempt to increase $l$ beyond $m$, the black hole located at $\left(r_{+}, 0\right)$ would attain a negative mass and thus become a naked singularity; at the other end of the segment $r=r_{+}$, the mass of the black hole would exceed the total mass of the solution. This is clearly an unphysical situation; thus we shall restrict ourselves to values of $l$ that are less than or equal to $m$.

When $l=0$, it is well known [5] that the conical singularity along $r=r_{+}$can also be removed by introducing an external magnetic field tuned to the appropriate strength; this was achieved by performing a dilatonic generalization of the Harrison transformation [13 on the $l=0$ case of our solution (3.7), (3.3) and (3.4). For general $l$, the transformation yields

$$
\begin{aligned}
& \mathrm{d} s^{2}= \Lambda^{\frac{2}{1+\alpha^{2}}}\left\{-\mathrm{d} t^{2}+\frac{\sum^{\frac{4}{1+\alpha^{2}}}}{\left[\Delta+\left(m^{2}+a^{2}-l^{2}\right) \sin ^{2} \theta\right]^{\frac{3-\alpha^{2}}{1+\alpha^{2}}}}\left(\frac{\mathrm{d} r^{2}}{\Delta}+\mathrm{d} \theta^{2}\right)\right\}+\frac{\Delta \sin ^{2} \theta}{\Lambda^{\frac{2}{1+\alpha^{2}}}} \mathrm{~d} \varphi^{2}, \\
& A_{\varphi}=-\frac{1}{\Sigma \Lambda^{\prime}}\left\{\frac{2}{\sqrt{1+\alpha^{2}}}\left[a \sin ^{2} \theta\left(m r-l^{2}\right)+l \Delta \cos \theta\right]\right. \\
&\left.\quad-\frac{B}{2}\left[\sin ^{2} \theta\left(r^{2}-a^{2}-l^{2}\right)^{2}+\Delta\left(a \sin ^{2} \theta-2 l \cos \theta\right)^{2}\right]\right\} \\
& \phi=-\frac{\alpha}{1+\alpha^{2}} \ln \Lambda^{\prime}
\end{aligned}
$$

where $\Delta$ and $\Sigma$ are the same as above,

$$
\begin{aligned}
& \Lambda^{\prime} \equiv \frac{1}{\Sigma}\left\{\Delta+a^{2} \sin ^{2} \theta-2 B \sqrt{1+\alpha^{2}}\left[a \sin ^{2} \theta\left(m r-l^{2}\right)+l \Delta \cos \theta\right]\right. \\
&\left.+\frac{1}{4} B^{2}\left(1+\alpha^{2}\right)\left[\sin ^{2} \theta\left(r^{2}-a^{2}-l^{2}\right)^{2}+\Delta\left(a \sin ^{2} \theta-2 l \cos \theta\right)^{2}\right]\right\},
\end{aligned}
$$

and $B$ is a new parameter governing the strength of the external magnetic field.

The values of $B$ that would remove the conical singularity along $r=r_{+}$are now

$$
B_{ \pm}=\frac{1}{\sqrt{1+\alpha^{2}}} \frac{a \pm \sqrt{m^{2}+a^{2}-l^{2}}}{m r_{+}-l^{2}} .
$$

Of these two possible values, $B_{+}$is unphysical as it remains non-zero in the limit of large $a$ [5]. Therefore, the only physically sensible $B$ that would remove the conical singularity along $r=r_{+}$is given by $B_{-}$.

\footnotetext{
IThe $a=0$ case, corresponding to the self-dual Taub-NUT solution, was precisely what Gross and Perry [1] and Sorkin [2] considered to obtain their Kaluza-Klein monopole solution.
} 
However, in contrast to the $l=0$ case [5], the conical singularities at the other axes of symmetry no longer vanish for this choice of $B$. If we assume that the coordinate $\varphi$ has its usual periodicity along the symmetry axis $r=r_{+}$, it can be shown that along the $\theta=0$ segment, there is a conical deficit of

$$
\delta_{(0)}=2 \pi\left\{1-\left[1-l\left(\frac{a-\sqrt{m^{2}+a^{2}-l^{2}}}{m r_{+}-l^{2}}\right)\right]^{-\frac{4}{1+\alpha^{2}}}\right\},
$$

corresponding to a cosmic string; whereas along the $\theta=\pi$ segment, there is a conical excess of

$$
\delta_{(\pi)}=2 \pi\left\{\left[1+l\left(\frac{a-\sqrt{m^{2}+a^{2}-l^{2}}}{m r_{+}-l^{2}}\right)\right]^{-\frac{4}{1+\alpha^{2}}}-1\right\},
$$

corresponding to a strut. Hence, for an unbalanced dihole, it is impossible to remove the conical singularities along the segments $\theta=0$ and $\theta=\pi$ simultaneously with that along $r=r_{+}$, by tuning the strength of the external magnetic field. Physically, this is expected due to the asymmetry in the distribution of charges among the two black holes.

Finally, we shall remark that instead of a magnetic dihole solution, an electric dihole solution can be obtained by dualizing the magnetic field strength tensor $F_{a b}$ via

$$
\phi^{\prime}=-\phi, \quad F^{\prime}{ }_{a b}=\frac{e^{-2 \alpha \phi}}{2} \epsilon_{a b c d} F^{c d} .
$$

Applying this transformation to the Harrison-transformed solution (3.12a) - (3.12d), we obtain a solution which describes an electrically charged dihole immersed in an external electric field. The corresponding gauge field is given by

$$
\begin{aligned}
A^{\prime}{ }_{t}= & (r-3 m) B \cos \theta-\frac{\sqrt{1+\alpha^{2}}}{2} B^{2}\left[m a \cos \theta\left(2+\sin ^{2} \theta\right)+l(r-3 m)\left(1+\cos ^{2} \theta\right)\right] \\
& +\frac{2}{\sqrt{1+\alpha^{2}}} \frac{m a \cos \theta-l(r-m)}{\Sigma}\left[1+\frac{\sqrt{1+\alpha^{2}}}{2} B\left(a \sin ^{2} \theta-2 l \cos \theta\right)\right]^{2},
\end{aligned}
$$

with all other components vanishing. In the special case $l=0$, the above expression reduces to that given by Chattaraputi et al. [14]. Note that $B$ now governs the strength of the external electric field. When $B=0$, (3.15) reduces to

$$
{A^{\prime}}_{t}=\frac{2}{\sqrt{1+\alpha^{2}}} \frac{m a \cos \theta-l(r-m)}{\Sigma},
$$

which clearly asymptotes to the gauge field generated by an electric point source, with charge $\frac{-2 l}{\sqrt{1+\alpha^{2}}}$, located at the origin. 


\section{D6-anti- $D 6$-brane configuration}

Now, for certain values of $\alpha$, the action (2.1) emerges from string theory when compactified down to four dimensions. In such cases the four-dimensional dihole solutions can be reinterpreted in terms of brane-anti-brane configurations in ten dimensions. Perhaps the most important example is when the Kaluza-Klein dipole is uplifted to ten dimensions, to describe a D6-anti-D6-brane configuration in Type IIA superstring theory [12].

In the string frame, the solution describing a pair of D6-branes with opposite but unbalanced magnetic charges, immersed in a non-trivial magnetic field, is given by (in standard string theory conventions [7])

$$
\begin{aligned}
& \mathrm{d} s^{2}=\Lambda^{\prime \frac{1}{2}}\left\{-\mathrm{d} t^{2}+\mathrm{d} x_{1}^{2}+\cdots+\mathrm{d} x_{6}^{2}+\Sigma\left(\frac{\mathrm{d} r^{2}}{\Delta}+\mathrm{d} \theta^{2}\right)\right\}+\frac{\Delta \sin ^{2} \theta}{\Lambda^{\prime} \frac{1}{2}} \mathrm{~d} \varphi^{2}, \\
& A_{\varphi}=-\frac{2}{\Sigma \Lambda^{\prime}}\left\{a \sin ^{2} \theta\left(m r-l^{2}\right)+l \Delta \cos \theta\right. \\
& \left.-\frac{B}{2}\left[\sin ^{2} \theta\left(r^{2}-a^{2}-l^{2}\right)^{2}+\Delta\left(a \sin ^{2} \theta-2 l \cos \theta\right)^{2}\right]\right\}, \\
& \phi=-\frac{3}{4} \ln \Lambda^{\prime}
\end{aligned}
$$

where we now have,

$$
\begin{aligned}
\Lambda^{\prime}=\frac{1}{\Sigma}\left\{\Delta+a^{2} \sin ^{2} \theta-4 B\left[a \sin ^{2} \theta\left(m r-l^{2}\right)+l \Delta \cos \theta\right]\right. \\
\left.\quad+B^{2}\left[\sin ^{2} \theta\left(r^{2}-a^{2}-l^{2}\right)^{2}+\Delta\left(a \sin ^{2} \theta-2 l \cos \theta\right)^{2}\right]\right\},
\end{aligned}
$$

with $\Delta$ and $\Sigma$ given in (3.6). The geometry of the individual $D 6$-branes, located at $(r, \theta)=$ $\left(r_{+}, 0\right)$ and $\left(r_{+}, \pi\right)$, can be recovered by performing the coordinate transformation (3.9) on the above solution. This solution contains, as a special case, the solution considered by Sen in [12].

As in the four-dimensional situation, if the external magnetic field is switched off by setting $B=0$, the branes coincide when $a=0$. In this limit, the solution simplifies to

$$
\begin{aligned}
\mathrm{d} s^{2}= & \left(\frac{r^{2}-2 m r+l^{2}}{r^{2}-l^{2}}\right)^{\frac{1}{2}}\left(-\mathrm{d} t^{2}+\mathrm{d} x_{1}^{2}+\cdots+\mathrm{d} x_{6}^{2}\right) \\
& +\left(\frac{r^{2}-l^{2}}{r^{2}-2 m r+l^{2}}\right)^{\frac{1}{2}}\left[\mathrm{~d} r^{2}+\left(r^{2}-2 m r+l^{2}\right)\left(\mathrm{d} \theta^{2}+\sin ^{2} \theta \mathrm{d} \varphi^{2}\right)\right] \\
A_{\varphi}= & -2 l \cos \theta \\
\phi= & -\frac{3}{4} \ln \left(\frac{r^{2}-2 m r+l^{2}}{r^{2}-l^{2}}\right)
\end{aligned}
$$


which describes a spherically symmetric fix-brane source carrying a monopole charge $l$. Recently, Brax et al. [7] presented a supergravity solution that corresponds to $N D p$-branes coinciding with $\bar{N}$ anti- $D p$-branes, with $N \neq \bar{N}$ in general. We will now establish a correspondence between our solution and theirs when $p=6$.

The solution of [7], after transforming to the string frame, is given by

$$
\begin{aligned}
\mathrm{d} s^{2} & =e^{\frac{\phi}{2}}\left\{e^{2 A(\tilde{r})}\left(-\mathrm{d} t^{2}+\mathrm{d} x_{1}^{2}+\cdots+\mathrm{d} x_{6}^{2}\right)+e^{2 B(\tilde{r})}\left[\mathrm{d} \tilde{r}^{2}+\tilde{r}^{2}\left(\mathrm{~d} \theta^{2}+\sin ^{2} \theta \mathrm{d} \varphi^{2}\right)\right]\right\}, \\
A_{t 1 \ldots 6} & =\sqrt{c_{2}^{2}-1} \frac{\sinh k h(\tilde{r})}{\cosh k h(\tilde{r})-c_{2} \sinh k h(\tilde{r})}, \\
\phi & =\frac{7}{16} c_{1} h(\tilde{r})-\frac{3}{4} \ln \left[\cosh k h(\tilde{r})-c_{2} \sinh k h(\tilde{r})\right],
\end{aligned}
$$

where

$$
\begin{aligned}
& A(\tilde{r}) \equiv-\frac{3}{64} c_{1} h(\tilde{r})-\frac{1}{16} \ln \left[\cosh k h(\tilde{r})-c_{2} \sinh k h(\tilde{r})\right], \\
& B(\tilde{r}) \equiv \ln \left(1-\frac{r_{0}^{2}}{\tilde{r}^{2}}\right)+\frac{21}{64} c_{1} h(\tilde{r})+\frac{7}{16} \ln \left[\cosh k h(\tilde{r})-c_{2} \sinh k h(\tilde{r})\right], \\
& h(\tilde{r}) \equiv \ln \left(\frac{\tilde{r}-r_{0}}{\tilde{r}+r_{0}}\right), \quad k \equiv \sqrt{4-\frac{7}{16} c_{1}^{2}} .
\end{aligned}
$$

In these expressions, $c_{1}, c_{2}$ and $r_{0}$ represent the three parameters of the solution, and the seven-form gauge field given by (4.3b) represents that of an electrically charged six-brane; for a magnetic six-brane, the corresponding dilaton field and gauge field can be obtained by performing an electromagnetic duality transformation on the above solution [7].

The parameter $c_{1}$ was argued in [0] to be related to the vacuum expectation value of the open string tachyon stretching between the D6- and anti-D6-branes. To establish the correspondence with our solution, we will set this parameter to zero. In addition, the other parameters are taken to be

$$
c_{2}=\frac{m}{\sqrt{m^{2}-l^{2}}} \quad \text { and } \quad r_{0}=\frac{\sqrt{m^{2}-l^{2}}}{2}
$$

Defining a new radial coordinate $r$ by

$$
\tilde{r}=\frac{1}{2}\left(r-m+\sqrt{r^{2}-2 m r+l^{2}}\right),
$$

*It is curious to note that the coincident limit of the four-dimensional dihole (3.7) solution is spherically symmetric only when $\alpha=\sqrt{3}$.

${ }^{\dagger}$ However, the physical significance of this parameter in four dimensions is still unclear. 
it can then be checked that the magnetic solution obtained by dualizing $4.3 \mathrm{a})-(4.3 \mathrm{c})$ is equivalent to $(4.2 \mathrm{a})-(4.2 \mathrm{~d})$. Thus, we see that our solution (4.1a) - (4.1c) contains, as a special case, the coincident $D 6$-anti- $D 6$-brane system of $[7]$.

\section{Conclusion}

In this paper, we have presented a solution-generating technique which was first used by Davidson and Gedalin [四 to generate black dihole solutions carrying equal but opposite charges. For any stationary, axisymmetric solution to the vacuum Einstein equations, we can find a corresponding static, axisymmetric solution to the Einstein-Maxwell-Dilaton equations via this technique.

As an application of the technique, we have constructed a new solution starting from the Kerr-NUT solution. A detailed analysis reveals that for $l \leq m$, the solution describes a pair of extremal dilatonic black holes lying on the symmetry axis. They carry unbalanced magnetic charges, with the net charge governed by the NUT-parameter $l$.

There are a few avenues for future research. Chattaraputi et al. 14 have recently found oppositely charged dihole solutions in $\mathrm{U}(1)^{4}$ gauge theory - a generalization of EinsteinMaxwell-Dilaton theory consisting of four abelian gauge fields and three scalar fields. When embedded in string or M-theory, these solutions describe a variety of intersecting braneantibrane configurations. It would be worth finding the corresponding solutions with unbalanced electric and/or magnetic charges.

It would also be of interest to find dihole solutions describing non-extremal black holes, as well as diholes in de Sitter and anti-de Sitter space. Another challenging problem is the construction of diholes in higher-dimensional Einstein-Maxwell-Dilaton theory. When embedded in string theory, these solutions would describe $D p$-anti- $D p$-brane configurations for $p \leq 5$.

\section{Acknowledgement}

We thank Roberto Emparan for constructive criticisms on an earlier version of the manuscript.

\section{References}

[1] D. J. Gross and M. J. Perry, "Magnetic monopoles in Kaluza-Klein theories," Nucl. Phys. B226, 29 (1983). 
[2] R. D. Sorkin, "Kaluza-Klein monopole," Phys. Rev. Lett. 51, 87 (1983).

[3] W. B. Bonnor, "An exact solution of the Einstein-Maxwell equations referring to a magnetic dipole," Z. Phys. 190, 444 (1966).

[4] A. Davidson and E. Gedalin, "Finite magnetic flux tube as a black and white dihole," Phys. Lett. B339, 304 (1994) gr-qc/9408006.

[5] R. Emparan, "Black diholes," Phys. Rev. D61, 104009 (2000) hep-th/9906160.

[6] M. Demiański and E. T. Newman, "A combined Kerr-NUT solution of the Einstein field equations," Bull. Acad. Polon. Sci. Ser. Math. Astron. Phys. 14, 653 (1966).

[7] P. Brax, G. Mandal and Y. Oz, "Supergravity description of non-BPS branes," Phys. Rev. D 63, 064008 (2001) hep-th/0005242.

[8] D. Kramer, H. Stephani, M. MacCallum and E. Herlt, "Exact solutions of Einstein's field equations," (Cambridge University Press, Cambridge, 1980).

[9] J. N. Islam, "Rotating fields in general relativity," (Cambridge University Press, Cambridge, 1985).

[10] G. W. Gibbons and K. Maeda, "Black holes and membranes in higher-dimensional theories with dilaton fields," Nucl. Phys. B298, 741 (1988).

[11] M. Carmeli, "Classical fields: general relativity and gauge theory," (John Wiley \& Sons, New York, 1982).

[12] A. Sen, "Strong coupling dynamics of branes from M-theory," JHEP 9710, 002 (1997) hep-th/9708002.

[13] F. Dowker, J. P. Gauntlett, D. A. Kastor and J. Traschen, "Pair creation of dilaton black holes," Phys. Rev. D49, 2909 (1994) hep-th/9309075.

[14] A. Chattaraputi, R. Emparan and A. Taormina, "Composite diholes and intersecting brane-antibrane configurations in string/M-theory," Nucl. Phys. B573, 291 (2000) hepth/9911007. 